\title{
[2.2]Paracyclophane derivatives containing tetrathiafulvalene moieties
}

\author{
Laura G. Sarbu ${ }^{1,2}$, Lucian G. Bahrin ${ }^{1,2}$, Peter G. Jones ${ }^{3}$, Lucian M. Birsa* ${ }^{*}, 2$ \\ and Henning Hopf ${ }^{*} 2$
}

\section{Letter}

\section{Address:}

${ }^{1}$ Department of Chemistry, "Al. I. Cuza” University of Iasi, 11 Carol I Bv., RO-700506 lasi, Romania, ${ }^{2}$ Institute of Organic Chemistry, Technical University of Braunschweig, Hagenring 30, D-38106 Braunschweig, Germany and ${ }^{3}$ Institute of Inorganic and Analytical Chemistry, Technical University of Braunschweig, Hagenring 30, D-38106 Braunschweig

\section{Email:}

Lucian M. Birsa* - lbirsa@uaic.ro; Henning Hopf* - h.hopf@tu-bs.de

* Corresponding author

Keywords:

dithiocarbamates; 1,3-dithiolium salts; [2.2]paracyclophane;

regioselective bromination; stereoisomers; tetrathiafulvalenes
Beilstein J. Org. Chem. 2015, 11, 1917-1921.

doi:10.3762/bjoc. 11.207

Received: 27 July 2015

Accepted: 24 September 2015

Published: 15 October 2015

This article is part of the Thematic Series "Tetrathiafulvalene chemistry".

Guest Editor: P. J. Skabara

(c) 2015 Sarbu et al; licensee Beilstein-Institut.

License and terms: see end of document.

\begin{abstract}
The synthesis of [2.2]paracyclophane derivatives containing tetrathiafulvalene units has been accomplished by the coupling reaction of 4-([2.2]paracyclophan-4-yl)-1,3-dithiol-2-thione in the presence of trimethylphosphite. The 1,3-dithiol-2-thione derivative was in turn synthesized by the regioselective bromination of 4-acetyl[2.2]paracyclophane, then through the corresponding dithiocarbamates and 1,3-dithiolium salts.
\end{abstract}

\section{Introduction}

Tetrathiafulvalene (TTF) and its derivatives have been extensively studied with respect to their applications as organic metals and superconductors [1,2]. These properties are a consequence of the $\pi$-donor properties of TTF and of its important intermolecular interactions in the solid state through extended $\pi$-orbitals. The design of new tetrathiafulvalene derivatives has targeted those systems where the intermolecular interactions between planar molecules are more effective and the solid-state architecture tends to be as stacks or layers with their long axes mutually parallel.

[2.2]Paracyclophane and its derivatives has been the subject of particular interest since their discovery, more than six decades ago [3-5]. Most of the unique properties of these cyclophanes are the result of the rigid framework and the short distance between the two aromatic rings within the [2.2]paracyclophane 
unit. Besides investigations of the geometry and of transannular interactions, special attention has been paid to the ability of these compounds to form charge-transfer complexes [6-9].

A recent report has appeared concerning the synthesis of a [2.2]paracyclophane doubly substituted by a dimeric tetrathiafulvalene in a pseudo-ortho substitution pattern [10]. This compound exhibited novel chiroptical properties. Prompted by these observations, we decided to investigate the synthesis of a tetrathiafulvalene that incorporates two [2.2]paracyclophane units.

\section{Results and Discussion}

The synthesis of [2.2]paracyclophanes containing a tetrathiafulvalene moiety, follows a general route that involves the synthesis of the corresponding [2.2]paracyclophane-substituted 1,3dithiol-2-ylium salts. These compounds are known to provide tetrathiafulvalenes under specific conditions [11-13]. The synthetic strategy for the incorporation of the 1,3-dithiol-2-ylium ring in the [2.2]paracyclophane framework involves a three-step reaction sequence, starting from 4-acetyl[2.2]paracyclophane (1) (Scheme 1).

The first step consists of the regioselective monobromination of 4-acetyl[2.2]paracyclophane (1) at the $\alpha$-position to the carbonyl group to form 2-bromo-1-([2.2]paracyclophan-4-yl)ethan-1one (2). The starting 4-acetyl[2.2]paracyclophane has been prepared according to the reported procedure [14]. In order to avoid the formation of side products during the bromination process [15], the synthesis of compound $\mathbf{2}$ has been accomplished using the molecular complex of bromine with dioxane [16]. However, although this reagent has often been employed in mild and selective bromination reactions [17], there are some difficulties concerning its isolation and handling [18]. In order to avoid these problems, we generated the reagent by mixing one equivalent of bromine with one equivalent of dioxane and adding dry dioxane to the resulting solid until the dissolution was complete. This solution was then added dropwise at room temperature to a solution of one equivalent of ketone $\mathbf{1}$ in dioxane, providing 2-bromo-1-([2.2]paracyclophan-4-yl)ethan1 -one (2) in $81 \%$ yield.

The reactions of $\alpha$-bromophenones with salts of dithiocarbamic acid, readily available from the reaction of secondary amines with carbon disulfide, represent an accessible route to variously substituted phenacyl carbodithioates [19]. Following this synthetic protocol, we obtained dithiocarbamates $4 \mathbf{a}-\mathbf{c}$ by reacting 2-bromo-1-([2.2]paracyclophan-4-yl)ethan-1-one (2) with sodium $N, N$-dimethyldithiocarbamate (3a), pyrrolidinium pyrolidine-1-carbodithioate (3b) and morpholinium morpholine-4carbodithioate $\mathbf{( 3 c )}$, respectively. These compounds were obtained as colorless crystals in $80 \%$ isolated yields. The structures of dithiocarbamates $\mathbf{4 a}-\mathbf{c}$ were inferred from their analytical and spectral data; thus the ${ }^{1} \mathrm{H}$ NMR spectra include the expected signals for the aliphatic hydrogen atoms from the dialkylamino groups, and the ${ }^{13} \mathrm{C}$ NMR spectra show the signals at ca. $196 \mathrm{ppm}$ attributable to the thiocarbonyl group. Finally, the structure of 2-([2.2]paracyclophan-4-yl)-2-oxoethyl- $N, N$ dimethyldithiocarbamate (4a) was unambiguously confirmed by X-ray crystallography (Figure 1). The molecular dimensions confirm the extensive $\mathrm{p}-\pi$ conjugation within the dithiocarbamate group; the $\mathrm{N}(1)-\mathrm{C}(19)$ bond length is 1.341(3) $\AA$, some $0.12 \AA$ shorter than $\mathrm{N}(1)-\mathrm{C}(20)$ and $\mathrm{N}(1)-\mathrm{C}(21)$, which are essentially $\sigma$-bonds. The cyclophane group displays the usual

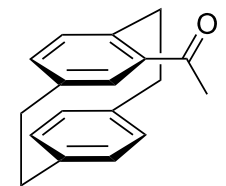

(rac)-1

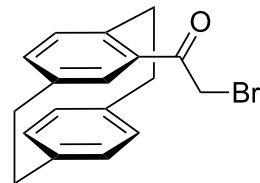

$(r a c)-2$

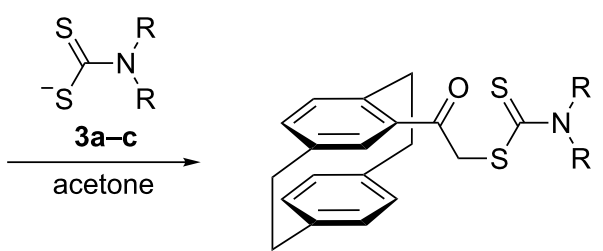

$(r a c)-4 a-c$

\begin{tabular}{crr}
\hline $\mathbf{3 , 4 , 5}$ & $\mathrm{R}$ & $\mathrm{R}$ \\
\hline a & $-\mathrm{CH}_{3}$ & $-\mathrm{CH}_{3}$ \\
b & \multicolumn{2}{c}{$-\left(\mathrm{CH}_{2}\right)_{5}-$} \\
c & \multicolumn{2}{c}{$-\left(\mathrm{CH}_{2}\right)_{2}-\mathrm{O}-\left(\mathrm{CH}_{2}\right)_{2-}^{-}$}
\end{tabular}

Scheme 1: Synthesis of 2-N,N-dialkylamino-4-([2.2]paracyclophan-4-yl)-1,3-dithiol-2-ylium perchlorates 5. 


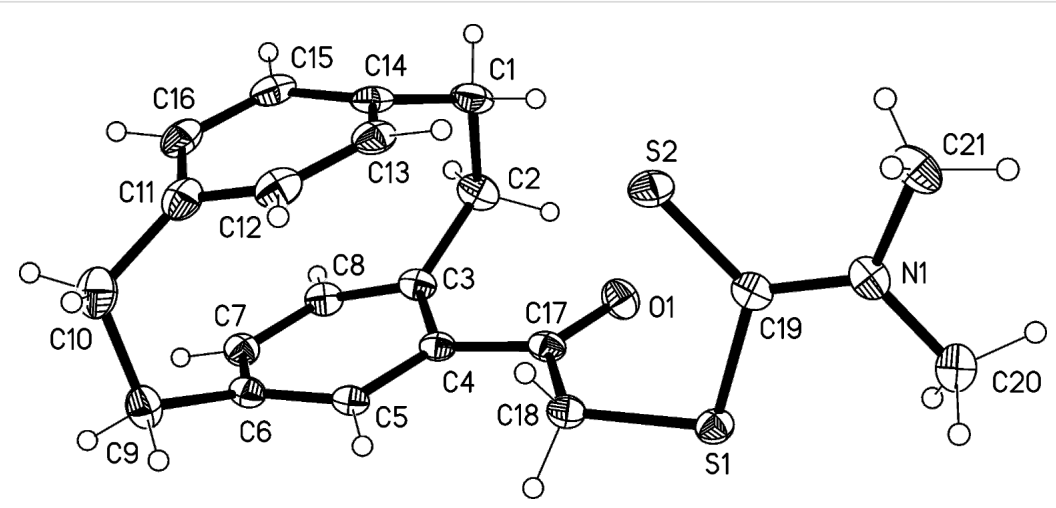

Figure 1: Molecular structure of compound 4a. Ellipsoids represent $30 \%$ probability levels. Selected molecular dimensions $\left(\AA{ }^{\circ}{ }^{\circ}\right)$ : $N(1)-C(19)$ 1.341(3), N(1)-C(20) 1.458(3), N(1)-C(21) 1.471(3), N(1)-C(19)-S(2) 123.19(17), N(1)-C(19)-S(1) 113.85(17), S(2)-C(19)-S(1) 122.89(13).

indicators of strain (e.g., lengthened bridge bonds and widened bridge angles, flattened boat conformations for the rings). The moieties $\mathrm{C} 3 / 4 / 5 / 17 / 18 / \mathrm{O} 1$ and $\mathrm{C} 19 / 20 / 21 / \mathrm{S} 1 / \mathrm{S} 2 / \mathrm{N} 1$ are to a good approximation planar and subtend an interplanar angle of $80^{\circ}$. Short intramolecular contacts $\mathrm{H} 13 \cdots \mathrm{S} 2 \quad 2.83 \AA$ and H2B $\cdots \mathrm{O} 12.29 \AA$ are observed. CCDC-1412356 contains the supplementary crystallographic data for compound $4 \mathbf{a}$ (see also Supporting Information File 1). These data can be obtained free of charge from the Cambridge Crystallographic Data Center via http://www.ccdc.cam.ac.uk/data request/cif.

The third step, the synthesis of 1,3-dithiol-2-ylium salts, consists of an acid-catalyzed cyclocondensation of phenacyl carbodithioates 4. Using a concentrated sulfuric acid-glacial acetic acid mixture $(1: 3, \mathrm{v} / \mathrm{v})[20]$, the cyclization of carbodithioates $\mathbf{4 a}-\mathbf{c}$ takes place under mild reaction conditions. After $10 \mathrm{~min}$ at $80{ }^{\circ} \mathrm{C}$ the homogeneous reaction mixture was cooled to room temperature and $70 \% \mathrm{HClO}_{4}$ and water were added. Filtration and recrystallization of the precipitate gave perchlorates $\mathbf{5 a}-\mathbf{c}$ as colorless crystals, in $58-81 \%$ yield (Scheme 1). The heterocyclization of carbodithioates 4 is supported by their spectral data. The IR spectra revealed the disappearance of the absorption band corresponding to the carbonyl group (ca. $1680 \mathrm{~cm}^{-1}$ ) and the presence of new, strong and broad absorption bands at $1000-1100 \mathrm{~cm}^{-1}$, corresponding to the perchlorate anion. The ${ }^{1} \mathrm{H}$ NMR spectra of 1,3-dithiol-2ylium perchlorates indicate the absence of the $\mathrm{AB}$ quartet pattern of the methylene hydrogen atoms from compounds 4 . The ${ }^{13} \mathrm{C}$ NMR spectra also confirm the successful synthesis of 1,3-dithiol-2-ylium salts 5 by the disappearance of the carbonyl and thiocarbonyl carbon atoms present in the carbodithioate spectra, as well as the appearance of a new signal at a very low field (ca. $180 \mathrm{ppm}$ ) which corresponds to the electron-deficient C-2 atom.

1,3-Dithiolium salts are valuable precursors for tetrathiafulvalenes. There are two main synthetic approaches that are mainly based on the exploitation of the electron-deficient character of the $\mathrm{C}-2$ carbon atom. One of these involves the conversion of 2-N,N-dialkylamino-1,3-dithiolium salts into the corresponding 2-unsubstituted 1,3-dithiolium salts, followed by the homocoupling of the carbene intermediate that is generated under basic conditions. Unfortunately, our attempts to synthesize the 2-unsubstituted derivative from 1,3-dithiolium perchlorates $\mathbf{5 a}-\mathbf{c}$ in the presence of tetrafluoroboric acid led to the degradation of the substrate. The second approach for the synthesis of tetrathiafulvalenes involves the desulfurative coupling of 1,3-dithiol-2-thiones in the presence of alkyl phosphites. 1,3Dithiol-2-thiones are easily available by the treatment of 1,3dithiol-2-ylium salts with sodium sulfide [21]. Thus, by treating perchlorates $5 \mathbf{5}-\mathbf{c}$ with sodium sulfide nonahydrate, at room temperature in ethanol, we obtained 4-([2.2]paracyclophan-4yl)-1,3-dithiol-2-thione (6) as a yellow solid in $41 \%$ isolated yield (Scheme 2).

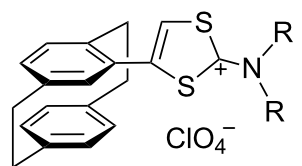

(rac)-5a-c
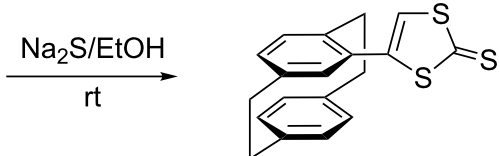

(rac)- 6

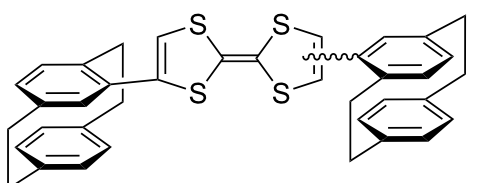

(rac)- 7 
Unexpectedly, the yield of 1,3-dithiol-2-thione 6 was found to be dependent on the nature of the dialkylamino substituent and to the volume of the solvent used. The best results were obtained by employing 2-( $N, N$-dimethylamino)-4-([2.2]paracyclophan-4-yl)-1,3-dithiol-2-ylium perchlorate (5a) as a substrate for this reaction. The formation of 1,3-dithiol-2-thione 6 is supported by the NMR and mass spectrometry data. Moreover, the structure of 1,3-dithiol-2-thione 6 was confirmed by $\mathrm{X}$-ray crystallography (Figure 2). The asymmetric unit contains two molecules, which differ in the orientation of the 1,3-dithiol2-thione group relative to the cyclophane; the torsion angle $\mathrm{C} 3-\mathrm{C} 4-\mathrm{C} 17-\mathrm{S} 3$ is $-45.9^{\circ}$ in the first molecule but $-142.4^{\circ}$ in the second. CCDC-1412357 contains the supplementary crystallographic data for compound 6 (see also Supporting Information File 1). These data can be obtained free of charge from The Cambridge Crystallographic Data Center via http:// www.ccdc.cam.ac.uk/data request/cif.

The desulfurative dimerization of 1,3-dithiol-2-thione 6 was effected by heating it with trimethyl phosphite at $100{ }^{\circ} \mathrm{C}$ (Scheme 2). Purification of the crude reaction mixture by column chromatography resulted in a mixture of inseparable isomeric tetrathiafulvalenes 7 in $17 \%$ yield. Since compound 6 already exhibits planar chirality (racemate $R_{\mathrm{p}} / S_{\mathrm{p}}$ ), the theoretical number of stereoisomers of the tetrathiafulvalene 7 should be six as follows: $\operatorname{cis}-\left(S_{\mathrm{p}}, S_{\mathrm{p}}\right)$ with $\operatorname{cis}-\left(R_{\mathrm{p}}, R_{\mathrm{p}}\right)$ and trans- $\left(S_{\mathrm{p}}, S_{\mathrm{p}}\right)$ with trans $-\left(R_{\mathrm{p}}, R_{\mathrm{p}}\right)$ for the parallel orientation of the ethano bridges, and the mesoforms cis- $\left(S_{\mathrm{p}}, R_{\mathrm{p}}\right)$ and trans- $\left(S_{\mathrm{p}}, R_{\mathrm{p}}\right)$ for the angular orientation of the ethano bridges. The NMR spectrum of the purified tetrathiafulvalene indicates the presence of 4 isomers, most probably the two pairs of racemates and the two mesoforms.

Although the separation of the isomers has not yet been successful, this synthetic pathway indicates that it is feasible to incorporate of [2.2]paracyclophane as an extended $\pi$-system onto a tetrathiafulvalene core. Further research will target a decrease in the theoretical number of isomers and their isolation in order to investigate their structural and chiroptical properties.

\section{Conclusion}

The synthesis of as yet inseparable isomeric tetrathiafulvalenes has been performed by desulfurative dimerization of a [2.2]paracyclophane-substituted trithione. The latter compound was obtained from the corresponding 1,3-dithiol-2-ylium cation. This was in turn synthesized through a three-step procedure, starting with the regioselective bromination of 4-acetyl[2.2]paracyclophane.

\section{Supporting Information}

\section{Supporting Information File 1}

Detailed experimental procedures, supplementary spectroscopic and X-ray data.

[http://www.beilstein-journals.org/bjoc/content/ supplementary/1860-5397-11-207-S1.pdf]

\section{Acknowledgements}

L.M.B. is indebted to the Alexander von Humboldt Foundation for a stay in Braunschweig. This work was supported by a grant of the Romanian National Authority for Scientific Research, CNDI-UEFISCDI, project number 51/2012.

\section{References}

1. Yamada, J. In TTF Chemistry Fundamentals and Applications of Tetrathiafulvalene; Yamada, J.; Sugimoto, T., Eds.; Kodansha and Springer: Tokyo, 2004.

2. Bendikov, M.; Wudl, F.; Perepichka, D. F. Chem. Rev. 2004, 104, 4891-4946. doi:10.1021/cr030666m

3. Brown, C. J.; Farthing, A. C. Nature 1949, 164, 915-916. doi:10.1038/164915b0

4. Cram, D. J.; Steinberg, H. J. Am. Chem. Soc. 1951, 73, 5691-5704. doi:10.1021/ja01156a059

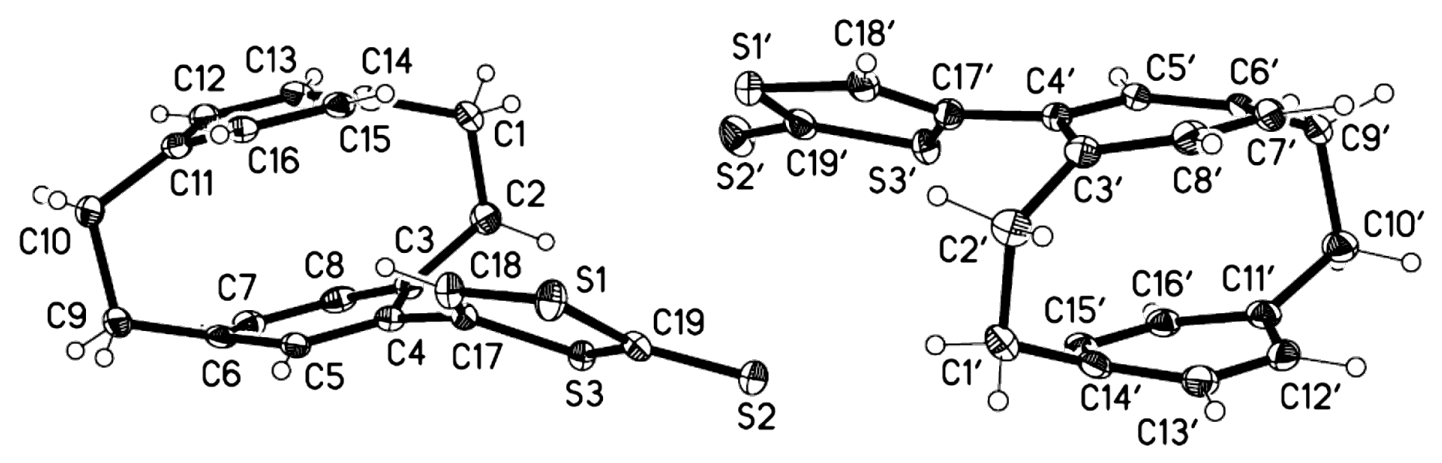

Figure 2: Molecular structure of compound 6 (two independent molecules). Ellipsoids represent $30 \%$ probability levels. 
5. Vögtle, F.; Neumann, P. Synthesis 1973, 85-103. doi:10.1055/s-1973-22137

6. Staab, H. A.; Knaus, G. H.; Henke, H.-E.; Krieger, C. Chem. Ber. 1983, 116, 2785-2807. doi:10.1002/cber.19831160807

7. Boekelheide, V. Top. Curr. Chem. 1983, 113, 87-143. doi:10.1007/3-540-12397-0_2

8. Hopf, H.; Marquard, C. Strain Release in Aromatic Molecules: The [2n] Cyclophanes. In Strain and its Implications in Organic Chemistry; de Meijere, A.; Blechert, S., Eds.; Kluwer: Dordrecht, 1989; pp 297-332. doi:10.1007/978-94-009-0929-8_21

9. Vögtle, F. Cyclophane Chemistry, Synthesis, Structure and Reactions; Wiley: Chichester, 1993; pp 71-111.

10. Kobayakawa, K.; Hasegawa, M.; Sasaki, H.; Endo, J.; Matsuzawa, H.; Sako, K.; Yoshida, J.; Mazaki, Y. Chem. - Asian J. 2014, 9, 2751-2754. doi:10.1002/asia.201402667

11. Narita, M.; Pittman, C. U., Jr. Synthesis 1976, 489-514. doi:10.1055/s-1976-24099

12. Schukat, G.; Richter, A. M.; Fanghänel, E. Sulfur Rep. 1987, 7, 155-231. doi:10.1080/01961778708082503

13. Schukat, G.; Fanghänel, E. Sulfur Rep. 1993, 14, 245-383. doi:10.1080/01961779308055019

14. Truesdale, E. A.; Cram, D. J. J. Org. Chem. 1980, 45, 3974-3981. doi:10.1021/j001308a005

15. Mamyrbekova, Z. A.; Soldatova, S. A.; Abelentsev, V. I.; Solov'eva, T. I.; Guryshev, V. N.; Soldatenkov, A. T. Pharm. Chem. J. 1994, 28, 198-202. doi:10.1007/BF02218999

16. Pasaribu, S. J.; Williams, L. R. Aust. J. Chem. 1973, 26, 1327-1331. doi:10.1071/CH9731327

17. Dombrovski, A. V. Russ. Chem. Rev. 1961, 30, 635-639. doi:10.1070/RC1961v030n12ABEH003011

18. Billimoria, J. D.; Maclagen, N. F. J. Chem. Soc. 1954, 3257-3262. doi:10.1039/jr9540003257

19. Lungu, N. C.; Sandu, I.; Chirita, P.; Birsa, M. L. Rev. Chim. (Bucharest, Rom.) 2013, 64, 697-700.

20. Sarbu, L. G.; Lungu, N. C.; Forna, N.; Birsa, M. L. Rev. Chim. (Bucharest, Rom.) 2013, 64, 1404-1407.

21. Birsa, M. L. Synth. Commun. 2003, 33, 3071-3076. doi:10.1081/SCC-120022483

\section{License and Terms}

This is an Open Access article under the terms of the Creative Commons Attribution License (http://creativecommons.org/licenses/by/2.0), which permits unrestricted use, distribution, and reproduction in any medium, provided the original work is properly cited.

The license is subject to the Beilstein Journal of Organic Chemistry terms and conditions: (http://www.beilstein-journals.org/bjoc)

The definitive version of this article is the electronic one which can be found at: $\underline{\text { doi:10.3762/bjoc. } 11.207}$ 